\title{
PARÁMETROS GENÉTICOS EN BERENJENA (Solanum melongena L.)
}

\section{GENETIC PARAMETERS IN EGGPLANT (Solanum melongena L.)}

\author{
Hermes Araméndiz-Tatis ${ }^{1}$, Carlos Cardona-Ayala ${ }^{2}$, Ender Correa-Álvarez ${ }^{3}$
}

\begin{abstract}
${ }^{1}$ I.A. Ph.D., Facultad de Ciencias Agrícolas, Universidad de Córdoba, Montería, Colombia, e-mail: haramendiz@hotmail. com; ${ }^{2}$ I.A., M.Sc., Facultad de Ciencias Agrícolas, Universidad de Córdoba, e-mail: ccardonaayala@yahoo.com; ${ }^{3}$ I.A. M.Sc., Corpoica C.I. Caribia. Sevilla (Mag.), e-mail: endermanz@hotmail.com
\end{abstract}

Rev. U.D.C.A Act. \& Div. Cient. 17(2): 361-369, Julio-Diciembre, 2014

\section{RESUMEN}

Parámetros genéticos de interés fueron estimados para el rendimiento de fruto y otros caracteres agronómicos en berenjena. El análisis de varianza reveló la existencia de variabilidad genética en todos los caracteres evaluados, a excepción del número de frutos por planta, en las líneas estudiadas. El coeficiente de variación genética osciló entre $7,33 \%$, para la longitud del fruto y, 39,47\%, para el número de semillas por fruto; los índices de variabilidad acusaron un rango entre 1,23, para la firmeza del fruto y, 2,70, para el número de semillas por fruto y, la heredabilidad en sentido amplio, fluctuó entre $60,45 \%$, para la firmeza del fruto y, $87,97 \%$, para el número de semillas por fruto. El mayor avance genético, se logró para la firmeza del fruto y número de ramas por planta, con $32,73 \%$ y $34,89 \%$, respectivamente, reflejando la efectividad de la selección sobre la variabilidad genética, presente en la población sometida a estudio.

Palabras clave: Varianza fenotípica, varianza genética, heredabilidad, índice de variación.

\section{SUMMARY}

Genetic parameters of interest were estimated for fruit yield and other agronomic characters in eggplant. Analysis of variance revealed the existence of genetic variability in all evaluated characters, except for the number of fruits per plant in the studied lines. The genetic variation coefficient ranged from $7.33 \%$ for fruit length and $39.47 \%$ for the number of seeds per fruit, the variability indices showed a range between 1.23 for firmness and 2.70 for seed number per fruit. The broad-sense heritability ranged from $60.45 \%$ for fruit firmness and $87.97 \%$ for the number of seeds per fruit. The greatest genetic advance was achieved for fruit firmness and number of branches per plant, with $32.73 \%$ and $34.89 \%$, respectively, reflecting the effectiveness of selection on genetic variability in the population studied.

Key words: Phenotypic variance, genetic variance, heritability, variation index.

\section{INTRODUCCIÓN}

La berenjena (Solanum melongena L.) es una hortaliza asiática, de gran importancia en el Caribe colombiano y de significativo arraigo en la culinaria, de los departamentos de Córdoba, Sucre y Bolívar, donde goza de aceptación en los diferentes estratos sociales, desde que fue introducida por las árabes, en el siglo XIX (Araméndiz et al. 2008b). De acuerdo a las estadísticas de Agronet (2013), en el 2006, fueron cultivadas 220 hectáreas, con rendimientos promedios de $7 \mathrm{t} \cdot$ ha $^{-1}$, que arrojaron una producción de 1.487 toneladas, guarismo inferior al del departamento del Valle del Cauca, que registró rendimientos de $38 \mathrm{t} \cdot \mathrm{ha}^{-1} \mathrm{y}$, a los de España y de México, principales exportadores, con rendimientos de 31,25 y $61,8 \mathrm{t} \cdot \mathrm{ha}^{-1}$, respectivamente (FAO, 2013), gracias al uso de cultivares mejorados, especialmente, híbridos de primera generación (Prohens \& Nuez, 2001).

El uso de semillas no certificadas y de sus propios cultivos representa una desventaja tecnológica de los agricultores del Caribe colombiano y una limitación para incursionar en los mercados internacionales, especialmente, a Estados Unidos de América, como lo han hecho México, Honduras y República Dominicana (FAO, 2013). Ante esta realidad, amerita aprovechar la variabilidad genética, para desarrollar cultivares adaptados a las condiciones ambientales, con características de frutos apetecibles por los consumidores nacionales e internacionales. 
La eficiencia de un programa de mejoramiento genético de cualquier especie depende de la variabilidad genética, presente en su acervo genético y la herencia de las características a mejorar, ya que ello es fundamental para realizar cambios significativos y así obtener cultivares mejorados, de mayor rendimiento y atributos agronómicos favorables. La existencia de variabilidad genética ha sido reportada en berenjena por investigadores, como Ramesh-Babu \& Patil (2008), quienes evaluaron 12 características cuantitativas en 90 cultivares de berenjena, resaltando la presencia de amplia variabilidad en todos los caracteres; Pirinç \& Pakyürek (2004) manifestaron la existencia de variación entre líneas derivadas de una población no mejorada en la longitud y diámetro del fruto, mas no para el rendimiento con respecto a la población parental; Muniappan et al. (2010) reportaron coeficientes de variación genético, entre $6,17 \%$, para floración hasta $45,39 \%$, para rendimiento de fruto por planta; Araméndiz et al. (2011) constataron la existencia de diversidad genética entre híbridos, cultivares mejorados y variedades locales e, igualmente, variabilidad genética entre los cultivares locales, para las variables agronómicas estudiadas.

La variación presente en un carácter métrico, se expresa como la varianza fenotípica, cuyos componentes corresponden a la varianza genotípica, que es la varianza de los valores genotípicos y se origina por las diferencias que existen entre genotipos; por lo que a mayor número de loci segregantes, mayor número de genotipos presentes en la población (Molina, 1992) y la varianza ambiental, que representa las desviaciones ambientales. En tanto, que la heredabilidad es uno de los parámetros importantes, dado que representa la importancia relativa de la variación heredable de la varianza fenotípica y es una propiedad de cada carácter y población característica (Moreno et al. 2002).

Considerando lo antes anotado, el objetivo de la investigación fue determinar la variabilidad genética, por medio de parámetros genéticos, en líneas obtenidas de una población de libre polinización de berenjena y el avance genético, a través de la selección.

\section{MATERIALES Y MÉTODOS}

Localización: El estudio, se realizó en el período comprendido entre junio de 2012 a febrero de 2013, en el municipio de Sampués - Sucre, finca la Florida, cuyas coordenadas geográficas corresponde a $9^{\circ} 10^{\prime} 32,3^{\prime \prime}$ latitud Norte y $75^{\circ} 22^{\prime} 57,2^{\prime \prime}$ longitud Oeste. El área de estudio presentó una temperatura promedio, durante el año, de $27,9^{\circ} \mathrm{C}$, una precipitación de $1100 \mathrm{~mm}$, humedad relativa del $88 \%$, brillo solar de 1860 horas/año y textura del suelo Franco arcillosoarenoso.
Material experimental: El material biológico usado en la investigación correspondió a 36 selecciones individuales, obtenidas de poblaciones de libre polinización y tres cultivares comerciales: Anasac, Long Purple y el híbrido comercial Galine.

Diseño experimental: El diseño experimental aplicado fue de Bloques Aumentados de Federer (1961), con nueve bloques. El proceso de aleatorización, se hizo en dos pasos: primero, las nueve repeticiones de cada uno de los tres tratamientos testigos (genotipos comunes), se asignaron completamente al azar, en forma independiente dentro de cada bloque, en las 63 unidades experimentales disponibles (parcelas); segundo, en las 36 unidades experimentales restantes de los nueve bloques, se asignaron al azar, los 36 nuevos tratamientos (genotipos nuevos); la aleatorización se hizo utilizando Excel 2010.

Cada genotipo, se sembró en dos surcos de $5 \mathrm{~m}$, con distancias entre surcos y plantas de $1,0 \mathrm{~m}$ y $1,0 \mathrm{~m}$, respectivamente, para 22 plantas por unidad experimental, 154 plantas por bloque y 1.386 plantas, en los nueve bloques aumentados.

Manejo agronómico: Se implementó el manejo integrado del cultivo, incorporando tecnologías apropiadas y buenas prácticas agrícolas, con el propósito de controlar variables exógenas, no contempladas en el estudio, que pudieran incidir en la expresión de los genotipos. La irrigación fue por aspersión, de acuerdo a las necesidades del cultivo.

Variables de respuesta: Se evaluaron ocho variables cuantitativas, considerando una parcela útil de cinco plantas, en plena competencia por tratamiento. Las variables cuantitativas correspondieron a: rendimiento (REND), número de frutos por planta (NFP), peso promedio de fruto (PPF), firmeza del fruto (FF), longitud del fruto (LF), diámetro del fruto (DF), número de ramas por planta (NRP) y número de semillas por fruto (NSF). El REND, se calculó mediante la sumatoria de 10 cosechas y se expresó en toneladas por hectárea $\left(\mathrm{t} \cdot \mathrm{ha}^{-1}\right)$ y la FF, se determinó mediante el uso del penetrómetro digital de frutas marca General Tools MFG, modelo FHT803 New York (USA).

Con la información registrada en campo, se realizaron análisis de varianza y prueba de comparación de medias para cada variable, con la ayuda del programa SAS versión 9.2 (SAS, 2008). Asimismo, fueron estimadas las varianzas fenotípica, genética, ambiental, heredabilidad en sentido amplio, coeficiente de variación genética en porcentaje e índice de variabilidad b y ganancia genética, mediante el uso del programa GENES versión Windows (2004.2.1), desarrollado por Cruz (2004).

Heredabilidad en sentido amplio: $h_{\mathrm{a}}^{2}=\frac{\sigma_{\mathrm{g}}^{2}}{\sigma_{\mathrm{p}}^{2}} \times 100$ 
La variabilidad en sentido amplio $\mathrm{h}_{\mathrm{a}}^{2}$ fue clasificada: baja $(<30 \%)$; moderada $(30 \%-60 \%)$ y alta $(>60 \%)$.

Coeficiente de variación genética: $\mathrm{CV}_{\mathrm{g}}=\frac{\sqrt{\sigma_{g}^{2}}}{\overline{\mathrm{x}}} \times 100$

Índice de variabilidad: $\mathrm{b}=\frac{\mathrm{CV}_{\mathrm{g}}}{\mathrm{CV}_{\mathrm{a}}}$

Avance genético $\left(\Delta_{g}\right): \mathrm{k} \times \sigma_{\mathrm{p}} \times \mathrm{h}_{\mathrm{a}}^{2}$

Donde:

$\mathrm{K}=$ Diferencial de selección al 5\% de intensidad de selección, equivalente a 2,06

$\sigma_{\mathrm{p}}=$ Desviación estándar fenotípica

$\mathrm{h}_{\mathrm{a}}^{2}=$ Heredabilidad en sentido amplio
El avance genético $\left(\Delta_{g}\right)$ en porcentaje: $\frac{\Delta_{g}}{\bar{x}} \times 100$

El rango del avance genético fue clasificado: bajo $(<10 \%)$, moderado $(10 \%-20 \%)$ y alto $(>20 \%)$.

\section{RESULTADOS Y DISCUSIÓN}

Diferencias altamente significativas y significativas (Tabla 1) fueron encontradas para todos los caracteres, con excepción de NFP en las líneas, resultados que concuerdan con los reportados por Suneetha \& Kathiria (2005); Sao \& Mehta (2010) y Chandra-Shekar et al. (2012), indicando la existencia de diferencias genética y posibilidades de selección de plantas, con atributos agronómicos favorables.

Tabla 1. Cuadrados medios para características agronómicas y de fruto, en cultivares de berenjena (Solanum melongena L.).

\begin{tabular}{|c|c|c|c|c|c|c|c|c|c|}
\hline Cultivar & G.L & 1 & 2 & 3 & 4 & 5 & 6 & 7 & 8 \\
\hline Bloques & 8 & $169,44^{* *}$ & $70,92^{* *}$ & $134,01^{\text {NS }}$ & $14,44^{* *}$ & $2,82^{*}$ & $0,008^{\text {NS }}$ & $0,58^{\text {NS }}$ & $59760,13^{\text {NS }}$ \\
\hline Testigos & 3 & $2996,51^{* *}$ & $577,95^{* *}$ & $104079,79^{* *}$ & $104,04^{* *}$ & $126,97^{* *}$ & $47,71^{* *}$ & $9,39^{* *}$ & $2568952,80^{* *}$ \\
\hline Líneas & 35 & $84,04^{* *}$ & $7,47^{\text {NS }}$ & $937,01^{* *}$ & $6,45^{* *}$ & $2,33^{*}$ & $0,37^{*}$ & $1,19^{* *}$ & $284707,20^{* *}$ \\
\hline Error & 16 & 24,60 & 12,99 & 221,26 & 2,94 & 1,00 & 0,10 & 0,38 & 41399,47 \\
\hline Total & 62 & 297,26 & 52,97 & 5848,24 & 13,81 & 8,59 & 2,58 & 1,40 & 347091,50 \\
\hline C.V. (\%) & & 8,52 & 13,00 & 5,83 & 15,97 & 5,61 & 5,13 & 10,81 & 18,41 \\
\hline
\end{tabular}

C.V. = coeficiente de variación; N.S. = ausencia de significancia; $*=$ significancia al 0,05 de probabilidad;

** = significancia al 0,01 de probabilidad

$1=$ Rendimiento, $2=$ Número de frutos por planta, $3=$ Peso promedio de frutos, $4=$ Firmeza del fruto, $5=$ Longitud del fruto, $6=$ Diámetro del fruto, $7=$ Número de ramas por planta, $8=$ Número de semillas por fruto.

Los coeficientes de variación (Tablas 1 y 2) resultaron bastante confiables, resaltando que la precisión experimental es comparable a la reportada por otros investigadores (Silva et al. 1999; Araméndiz et al. 2011; Kumar et al. 2013). El valor más bajo correspondió al carácter DF $(4,81)$ y el más alto al NSF $(28,32)$, lo que, para este último, puede obedecer a respuestas variables de los genotipos de naturaleza bioquímica y fisiológica, relacionadas con contenidos de proteína, azúcar, almidón, hormonas y tasa de respiración, que afectan el desarrollo del ovario, después de la polinización, como lo sostienen Ofosu-Anim et al. (2006).

Las diferencias significativas entre los testigos y en las líneas para el PPF refleja el contraste genético, entre una población homogénea heterocigota y una heterogénea homocigota y posibilita la selección de genotipos con genes deseables, como lo sostienen Kumar et al. (2013) y que coincide con los resultados reportados por Bogoev \& Kostadinov (2011).
El PPP en los testigos osciló entre 106g, para el cultivar Long Purple y 295g, para el híbrido Galine (Tabla 3), con promedio de 198,34g (Tabla 2), en tanto que para las líneas varió entre 198g, en el genotipo CO38 y, 364g, en PH06 (Tabla 3), con un promedio de 297,28g (Tabla 2), lo cual, permite seleccionar plantas con frutos, de acuerdo a las exigencias del mercado.

Los parámetros genéticos estimados para el PPF destacan superioridad de la varianza genética con respecto a la ambiental, al igual que alta heredabilidad y coeficiente de variación genética, como fue reportado por Kumar et al. (2013), lo que favorece la selección de líneas con mejor correlación con el fenotipo, como C010, C015, C029, P01, P03, P06, P09, PH05, PH13, PH14, PH16, PH17, PH18, PH24, PH25 y PH36 (Tabla 3), dada la poca influencia del ambiente en la identificación de genotipos más destacados y avance genético, de 20,48\% (Tabla 2), ganancia que supera las obtenidas 
Tabla 2. Valores medios, rangos y parámetros genéticos estimados para ocho características agronómicas y de fruto en berenjena (Solanum melongena L.).

\begin{tabular}{|c|c|c|c|c|c|c|c|c|c|c|c|c|c|}
\hline VARIABLES & $\overline{X_{G}}$ & Rango & $\overline{X_{T}}$ & $\overline{X_{L}}$ & $\begin{array}{c}C V_{T} \\
(\%)\end{array}$ & $\begin{array}{l}C V_{L} \\
(\%)\end{array}$ & $\sigma_{f}^{2}$ & $\sigma_{a}^{2}$ & $\sigma_{g}^{2}$ & $\begin{array}{l}h_{a}^{2} \\
(\%)\end{array}$ & $\begin{array}{c}C V_{g} \\
(\%)^{-}\end{array}$ & b & $\underset{(\%)}{\Delta}$ \\
\hline Rendimiento & 58,17 & $36,31-88,23$ & 46,31 & 67,09 & 10,70 & 7,39 & 113,90 & 24,60 & 89,29 & 78,39 & 14,09 & 1,90 & 25,55 \\
\hline Número de frutos/planta & 27,72 & $18,00-40,00$ & 30,04 & 25,98 & 12,00 & 13,87 & 9,20 & 13,00 & 0 & 0 & 0 & 0 & 0 \\
\hline Firmeza & 10,74 & $6,58-18,52$ & 11,32 & 10,30 & 15,15 & 16,64 & 7,44 & 2,94 & 4,50 & 60,45 & 20,58 & 1,23 & 32,73 \\
\hline Longitud del fruto & 17,82 & $12,97-22,66$ & 15,89 & 19,26 & 6,28 & 5,18 & 2,99 & 0,99 & 1,99 & 66,64 & 7,33 & 1,41 & 12,20 \\
\hline Número de ramas/planta & 5,72 & $4,45-9,32$ & 4,98 & 6,28 & 12,10 & 9,58 & 1,55 & 0,36 & 1,19 & 76,61 & 17,35 & 1,81 & 34,89 \\
\hline Número de semillas/fruto & 1104,66 & $\begin{array}{l}531,00- \\
2858,00\end{array}$ & 718,27 & 1394,44 & 28,32 & 14,52 & 344340,1 & 41399,5 & 302940,5 & 87,97 & 39,47 & 2,70 & 24,11 \\
\hline
\end{tabular}

$\overline{X_{G}}=$ media general; $\overline{X_{T}}=$ media de testigos; $\overline{X_{L}}=$ media de líneas; $C V_{T}(\%)=$ coeficiente de variación de testigos; $C V_{L}(\%)=$ coeficiente de variación de líneas; $\sigma_{f}^{2}=$ varianza fenotípica; $\sigma_{a}^{2}=$ varianza ambiental; $\sigma_{g}^{2}=$ varianza genética; $h_{a}^{2}(\%)=$ heredabildad en sentido amplio; (\%) $C V_{g}=$ coeficiente de variación genética; b $=$ índice de variabilidad; $\Delta_{g}(\%)=$ avance genético.

por Prabhu et al. (2009b). A este respecto, frutos más pequeños son menos pesados y más susceptibles a la pérdida de agua, debido a una mayor proporción del cáliz cubriendo el fruto, lo que afecta el tiempo de conservación y calidad del mismo (Díaz-Pérez, 1998); por lo que frutos comprendidos entre los $250 \mathrm{~g}$ y $300 \mathrm{~g}$ son ideales para el mercado en fresco y asimismo, tienen un efecto directo en el rendimiento, como lo reportaron Muniappan et al. (2010) y Araméndiz et al. (2010).

La LF registró, a nivel de testigos y de líneas, diferencias genéticas entre los genotipos, circunstancia que concuerda con lo expuesto por Pirinç \& Pakyürek (2004) y Kumar et al. (2013), entre y dentro de poblaciones de berenjena.

El comportamiento de los testigos, para LF, registró un rango entre 12,97 y $19,60 \mathrm{~cm}$ (Tabla 3), con un promedio de $15,89 \mathrm{~cm}$ (Tabla 2); en tanto, que en las líneas, sus valores fluctuaron entre 15,0 y $22,66 \mathrm{~cm}$ (Tabla 3), con un promedio de $19,26 \mathrm{~cm}$ (Tabla 2), calificados como grandes, de acuerdo a las normas del ICONTEC (2004), para esta hortaliza.

La varianza genética estimada fue de superior magnitud con respecto a la varianza ambiental; asimismo, la alta heredabilidad, el coeficiente de variación genética e índice de variabilidad, confirman la existencia de variabilidad genética en la LF e indica el alcance de mejorar este carácter, como ha sido reportado por Kumar et al. (2013), permitiendo un avance genético moderado, como respuesta a la selección (Tabla 2). Alta heredabilidad y poco avance genético fue reportado, previamente, por Prabhu et al. (2009a) y Singh et al. (2003), debido a la presencia de genes con acción génica aditiva y no aditiva, como lo sostienen Prabhu et al. (2009b) y Chandra-Shekar et al. (2012), ya que la fracción no aditiva no es heredable y limita las posibilidades de la selección más eficiente de LF, entre 18 y $20 \mathrm{~cm}$, longitudes apetecidas en el mercado.

El DF, en los testigos, exhibió diferencias genéticas, a nivel de testigos y de líneas, mostrando coherencia con los resultados anotados por Pirinç \& Pakyürek (2004) y MuñozFalcón et al. (2009).

Los cultivares comerciales acusaron, para el DF, rangos entre 3,17 y $8,47 \mathrm{~cm}$ (Tabla 3), con un valor medio de $5,61 \mathrm{~cm}$ (Tabla 2), en tanto, que las líneas registraron rango entre 4,95 y $9,01 \mathrm{~cm}$ (Tabla 2), con promedio de $6,58 \mathrm{~cm}$ (Tabla 2 ). Estas diferencias están asociadas con el tipo de fruto, puesto que los delgados corresponden a los cultivares tipo serpentinum, que se caracterizan por ser de menor diámetro, mientras que los tipo esculentum, poseen forma aperada y mayor diámetro (Araméndiz et al. 2008b).

Las magnitudes de los componentes de la varianza fenotípica acusaron superioridad de la varianza genética, con respecto a la ambiental, alta heredabilidad $(76,36 \%)$, coeficiente de variación genética $(8,64 \%)$ e índice de variación $(b=1,79)$, que sustentan la variabilidad genética existente entre los genotipos, con avance genético moderado (Tabla 2), a causa del efecto del ambiente, en la expresión fenotípica del DF, por lo que la selección directa es menos efectiva, como lo destaca Singh et al. (2003).

El NFP registró diferencias genéticas únicamente entre los testigos y falta de significancia entre las líneas, resaltando la falta de variabilidad genética, entre las mismas (Tabla 1). En los testigos, el NFP acusó rango entre 23 y 40 (Tabla 3,) con una media de 30,04 (Tabla 2), sobresaliendo el cultivar 
Tabla 3. Valores promedios de nueve características agronómicas en testigos comerciales de berenjena (Solanum melongena L.).

\begin{tabular}{|c|c|c|c|c|c|c|c|c|}
\hline Cultivar & $1\left(\mathrm{t} \mathrm{ha}^{-1}\right)$ & $2(\#)$ & $3(\mathrm{~g})$ & $4\left(\mathrm{kgf} / \mathrm{cm}^{2}\right)$ & $5(\mathrm{~cm})$ & $6(\mathrm{~cm})$ & $7(\#)$ & $8(\#)$ \\
\hline Galine & 58,76 & 23 & 295 & 15,51 & 12,97 & 8,47 & 4,87 & 710 \\
\hline Anasac & 43,87 & 26 & 193 & 11,04 & 15,11 & 5,20 & 5,36 & 912 \\
\hline Long Purple & 36,31 & 40 & 106 & 7,41 & 19,60 & 3.17 & 4,70 & 531 \\
\hline $\mathrm{C} 010$ & 53,20 & 22 & 258 & 9,78 & 17,66 & 6,48 & 6,25 & 659 \\
\hline C015 & 55,47 & 23 & 257 & 7,38 & 19,66 & 6,18 & 6,85 & 663 \\
\hline C022 & 67,87 & 31 & 242 & 7,38 & 22,66 & 6,08 & 6,25 & 1338 \\
\hline C029 & 59,92 & 25 & 263 & 9,98 & 20,96 & 6,08 & 7,25 & 1438 \\
\hline $\mathrm{CO38}$ & 41,12 & 25 & 198 & 12,15 & 16,56 & 4,95 & 5,62 & 971 \\
\hline P01 & 64,88 & 24 & 284 & 13,85 & 17,42 & 6,38 & 4,45 & 1178 \\
\hline P03 & 62,48 & 28 & 296 & 14,92 & 20,22 & 6,41 & 9,32 & 1322 \\
\hline P04 & 82,42 & 26 & 314 & 14,35 & 20,56 & 6,45 & 6,02 & 1314 \\
\hline P05 & 82,43 & 27 & 348 & 9,68 & 18,32 & 6,81 & 5,78 & 1401 \\
\hline P06 & 68,78 & 28 & 286 & 11,72 & 15,19 & 6,21 & 5,55 & 7,81 \\
\hline P08 & 73,27 & 26 & 303 & 10,95 & 19,66 & 6,45 & 6,02 & 1836 \\
\hline P09 & 72,03 & 26 & 298 & 9,42 & 16,69 & 6,31 & 5,55 & 983 \\
\hline P10 & 56,85 & 27 & 310 & 11,18 & 18,52 & 6,61 & 4,78 & 1034 \\
\hline PH04 & 60,43 & 23 & 361 & 11,12 & 17,12 & 9,01 & 9,32 & 1249 \\
\hline PH05 & 61,17 & 31 & 281 & 6,08 & 19,49 & 7,01 & 4,48 & 2858 \\
\hline PH06 & 82,12 & 21 & 364 & 9,45 & 19,56 & 6,95 & 8,02 & 2387 \\
\hline PH07 & 85,32 & 28 & 311 & 13,25 & 18,56 & 6,35 & 4,62 & 1993 \\
\hline PH09 & 62,83 & 18 & 341 & 10,95 & 20,02 & 5,98 & 4,75 & 1070 \\
\hline PH10 & 56,85 & 19 & 340 & 11,32 & 20,12 & 6,65 & 7,18 & 603 \\
\hline PH11 & 66,02 & 28 & 317 & 11,88 & 19,29 & 6,51 & 5,18 & 1013 \\
\hline PH12 & 80,63 & 25 & 334 & 5,48 & 20,62 & 6,71 & 6,78 & 888 \\
\hline PH13 & 61,47 & 29 & 282 & 9,18 & 18,79 & 6,61 & 5,48 & 1956 \\
\hline PH14 & 61,82 & 30 & 253 & 7,78 & 19,19 & 6,81 & 5,18 & 2173 \\
\hline PH16 & 65,62 & 28 & 265 & 10,85 & 18,86 & 6,05 & 6,62 & 2242 \\
\hline PH17 & 61,28 & 26 & 299 & 7,62 & 22,02 & 6,41 & 7,62 & 711 \\
\hline PH18 & 50,48 & 21 & 255 & 14,02 & 17,99 & 7,91 & 7,55 & 2459 \\
\hline PH21 & 79,27 & 22 & 357 & 9,75 & 18,86 & 6,95 & 6,62 & 919 \\
\hline PH23 & 63,90 & 26 & 300 & 18,52 & 20,32 & 6,85 & 5,58 & 1019 \\
\hline PH24 & 69,28 & 25 & 296 & 8,92 & 17,19 & 5,61 & 7,85 & 1519 \\
\hline $\mathrm{PH} 25$ & 64,03 & 23 & 290 & 6,58 & 18,96 & 7,01 & 5,38 & 1534 \\
\hline PH26 & 88,23 & 28 & 326 & 9,25 & 19,42 & 7,48 & 6,45 & 2540 \\
\hline PH27 & 56,60 & 23 & 284 & 7,72 & 20,42 & 6,25 & 8,18 & 1357 \\
\hline PH28 & 79,91 & 21 & 298 & 8,42 & 22,42 & 6,75 & 5,58 & 951 \\
\hline PH35 & 57,28 & 25 & 305 & 8,02 & 19,96 & 6,41 & 5,62 & 1182 \\
\hline PH36 & 66,98 & 28 & 266 & 10,65 & 20,12 & 6,48 & 6,45 & 1244 \\
\hline S006 & 73,77 & 27 & 302 & 11,35 & 22,06 & 6,65 & 6,02 & 1402 \\
\hline
\end{tabular}

$1=$ rendimiento $2=$ número de frutos por planta, $3=$ peso promedio de frutos, $4=$ firmeza del fruto, $5=$ longitud del fruto, $6=$ diámetro del fruto, $7=$ número de ramas por planta, $8=$ número de semillas por fruto. 
Long Purple, con 40 frutos y peso de 106g (Tabla 3). De igual manera, en las líneas, el NFP registró variación entre 18,00 y 31,00, con un promedio de 25,98 frutos (Tabla 3). El genotipo con mayor NFP fue PH05, con 31,0, en tanto que PH09 registró 18,00; resultados similares fueron exhibidos por Polignano et al. (2010) y superiores a los revelados por Muñoz-Falcón et al. (2009), en cultivares comerciales, cuyo rango fue de 5,8 a 13,0 .

La presencia de varianza genética negativa para el NFP (Tabla 2), se explica de acuerdo a Hallauer \& Miranda (1985), a inadecuada técnica experimental o tamaño de muestra utilizada en la investigación; respuestas similares han sido reportadas por García et al. (2006), en chile jalapeño.

La existencia de diferencias significativas entre testigos y líneas para el número de ramas por planta, revela variabilidad genética entre los cultivares en estudio y posibilidades de éxitos, a través de la selección. Resultados similares fueron manifestados por Prabhu et al. (2009a); Prabhu et al. (2009b); Muñoz-Falcón et al. (2009) y Muniappan et al. (2010).

El rango del NRP, para los testigos, osciló entre 4,70 y 5,36 (Tabla 3), con promedio de 4,98 (Tabla 2), sobresaliendo el cultivar Long Purple, con 4,70 y Anasac, con 5,36. En las líneas, la variación fluctuó entre 4,45 y 9,32, con promedio de 4,98 NRP; los genotipos PH04 y P03 presentaron el número máximo, con 9,32 y el genotipo P01, un valor de 4,45 (Tabla 3).

La varianza genética para NRP resultó superior a la varianza ambiental y acusó alta heredabilidad. El coeficiente de variación genética e índice de variación corroboran apreciables niveles de variabilidad genética, como fue reportado por $\mathrm{Mu}-$ niappan et al. (2010) y el porqué de la magnitud del avance genético (Tabla 2). Estos resultados son similares a los encontrados por Chandra-Shekar et al. (2012) y, por las acciones génicas aditiva y no aditiva, en el control de este carácter (Kumar et al. 2013), es posible mejorar indirectamente el REND, por sus efectos directos sobre éste (Prabhu \& Natarajan, 2008; Sao \& Mehta, 2010).

El NSF reveló diferencias genéticas entre testigos y líneas, lo que posibilita la selección de cultivares con ventajas para su consumo en fresco y agroindustria, ya que las semillas tienen incidencia en la calidad del fruto, puesto que contribuyen a incrementar las saponinas y las solaninas en la pulpa, generando un sabor amargo, no deseado por el consumidor (Donzella et al. 2000).

Los testigos presentaron rangos entre 531 y 912 NSF (Tabla 3 ), con un promedio de 718,27 semillas (Tabla 2), correspondiendo a la variedad Anasac, 912 semillas y Long Purple, 531 semillas; en tanto, que las líneas expresaron varia- ción entre 603,00 y 2858,00 semillas, con un promedio de 1394,44 semillas, confirmando, una vez más, la variabilidad genética (Tabla 3). Se destaca por su alto número de semillas la líneas PH05, con 2858, contrastando con PH10, con 603. Los valores de este carácter superan los reportados previamente por Rylski et al. (1984) y Araméndiz et al. (2008a), en otras poblaciones.

La varianza genética estimada para NSF resultó de magnitud apreciable con relación a la varianza ambiental y explica la alta heredabilidad encontrada para este carácter. Asimismo, el coeficiente de variación genética e índice de variación confirman la variabilidad genética y el alto avance logrado, a través de la selección por la acción génica aditiva, que controla este carácter (Tabla 2), lo que permite seleccionar genotipos con menor NSF.

El análisis de varianza reveló diferencias significativas entre testigos y líneas para FF, lo que se sustenta en la variación genética existente entre los cultivares y concuerda con lo manifestado por Gajewski \& Arasimowicz (2004) y Singh et al. (2003), en cultivares de berenjena.

La FF registró en los testigos un rango, entre $7,41 \mathrm{kgf} / \mathrm{cm}^{2}$ y $15,51 \mathrm{kgf} / \mathrm{cm}^{2}$ (Tabla 3), siendo el híbrido Galine, el de mayor resistencia, con $15,51 \mathrm{kgf} / \mathrm{cm}^{2}$ (Tabla 2). En las líneas osciló entre $6,58 \mathrm{kgf} / \mathrm{cm}^{2}$ y $18,52 \mathrm{kgf} / \mathrm{cm}^{2}$, correspondiendo el segundo valor de firmeza al genotipo PH23 (Tabla 3), lo que es una ventaja agronómica en el transporte, como lo sostienen Araméndiz et al. (2011) y ello es atribuido a las características físicas de la pulpa (Gajewski \& Arasimowicz, 2004; Gajewski et al. 2009), por el papel del calcio en la estructura y por la función de las paredes celulares que interactúa con sustancia pépticas al interior de la pared celular, para formar pectato de calcio, dándole estabilidad a las paredes celulares.

La varianza genética resultó de mayor magnitud que la varianza ambiental para la firmeza del fruto, la que asociada a heredabilidad moderada, coeficiente de variación genética e índice de variabilidad, confirman la existencia de variabilidad genética, para este carácter y explica el porqué del avance genético del 32,73\%. Esto puede obedecer al rol de la acción génica aditiva y al reducido efecto ambiental en la expresión del fenotipo y, por lo tanto, sugiere una selección más efectiva de frutos, con mayor firmeza en los genotipos PH 23, P03, P04 y PH18 (Singh et al. 2003).

Tal como se desprende en el análisis de varianza (Tabla 1), a nivel de testigos, el híbrido Galine, con $58,76 \mathrm{t} \cdot \mathrm{ha}^{-1}$, fue superior en rendimiento a los cultivares Anasac y Long Purple, que acusaron valores de $43,87 \mathrm{t} \cdot \mathrm{ha}^{-1}$ y $36,31 \mathrm{t} \cdot \mathrm{ha}^{-1}$, respectivamente (Tabla 3); ello obedece, a la mayor heterosis en el cultivar Galine y a la relación directa existente entre el PPF, DF y 
NFP, con respecto al REND, como lo reportaron Araméndiz et al. (2010); Prabhu \& Natarajan (2008); Muniappan et al. (2010). Los cultivares Anasac y Long Purple arrojaron bajo REND, a causa de un menor PPF y DF, lo que indica que frutos de menor PPF y DF no tributan a un mayor REND, como lo sostienen Mazzucato et al. (2010); sin embargo, poseen ventaja en el mercado para su consumo, como encurtido (Araméndiz et al. 2011).

El REND de las líneas registró un rango entre $41,12 \mathrm{t} \cdot \mathrm{ha}^{-1} \mathrm{y}$ $88,23 \mathrm{t} \cdot \mathrm{ha}^{-1}$, con un valor medio de $67,06 \mathrm{t} \cdot \mathrm{ha}^{-1}$ (Tabla 3 ), el cual, resultó superior a los testigos comerciales, lo que indica que las diferencias detectadas en el análisis de varianza tienen un fuerte componente genético y selección de líneas con potencial de rendimiento, superior a las $80 \mathrm{t} \cdot \mathrm{ha} \mathrm{H}^{-1}$.

Los parámetros genéticos estimados revelan ventaja de la varianza genética con respecto a la ambiental y heredabilidad en sentido amplio, de 78,39\%, que asociada con el coeficiente de variación genético $(14,09)$ e índice de variabilidad b $(1,90)$, ratifican la existencia de variabilidad genética y una fuerte correlación entre el fenotipo y el genotipo, lo que permite seleccionar líneas con genes deseables, para transmitir a sus descendencia; hecho que concuerda con las investigaciones adelantadas por Prabhu et al. (2009a), en berenjena y Saeed et al. (2007), en tomate.

Las estimativas de parámetros genéticos permiten concluir la existencia de apreciable variabilidad genética aprovechable, a través de la selección y lograr avances genéticos relevantes, especialmente en la FF, que juega un rol importante en la calidad y NRP, en el REND.

Conflicto de intereses: El manuscrito fue preparado y revisado con la participación de todos los autores, quienes declaramos que no existe ningún conflicto de intereses, que ponga en riesgo la validez de los resultados presentados. Financiación: Este estudio fue financiado con fondos de la Universidad de Córdoba.

\section{BIBLIOGRAFÍA}

1. AGRONET. 2013. Sistema de Estadísticas Agropecuarias - SEA. Área, producción y rendimiento. Principales Departamentos Productores de Berenjena' Ordenados por Rendimiento, 2006. Disponible deasde Internet en: http://www.agronet.gov.co (con acceso 05/05/2013).

2. ARAMÉNDIZ-TATIS, H.; SUDRÉ, C.P.; GONÇALVES, L.S.A.; RODRIGUES, R. 2011. Potencial agronômico e divergência genética entre genótipos de berinjela nas condições do Caribe Colombiano. Hort. Bras. 29(2):174-180.
3. ARAMÉNDIZ-TATIS, H.; ESPITIA, M.; CARDONA, C. 2010. Análisis de sendero en berenjena (Solanum melongena L.). Rev. U.D.C.A. Act. \& Div. Cient. 13(1):115-123.

4. ARAMÉNDIZ-TATIS, H.; CARDONA, C.; PÉREZ, D. 2008a. Hibridación artificial en berenjena (Solanum melongena L.): Efecto sobre la producción de frutos y semillas. Rev. U.D.C.A. Act. \& Div. Cient. 11(2): 121-130.

5. ARAMÉNDIZ, H.; CARDONA, C.; JARMA, A.; ESPITIA, M. 2008b. El cultivo de la Berenjena (Solanum melongena L.). $1^{\mathrm{a}}$ ed. Bogotá, Ed. Produmedios. 152p.

6. BOGOEV, G.; KOSTADINOV, K. 2011. Comparative testing of varieties eggplant, kept for intermediate agrarian production. Trakia J. Sci. 9(4):39-42.

7. CRUZ, C. 2004. Programa genes. Versão Windows. Aplicativo computacional em genética e estatística. Universidade Federal de Viçosa. Disponible desde Internet en: http:///www.ufv.br/dbg/genes/genes.htm (con acceso 17/07/2007).

8. CHANDRA-SHEKAR, K.; ASHOK, P.; SASIKALA, K. 2012. Studies on heritability and multivariate analysis in brinjal (Solanum melongena L.). Veg. Crops Res. Bull. 76(1):79-88.

9. DÍAZ-PÉREZ, J.C. 1998. Transpiration rates in eggplant fruit as affected by fruit and calyx size. Postharvest Biol. Techn. 13(1):45-49.

10. DONZELLA, G.; SPENA, A.; ROTINO, G. 2000. Transgenic parthenocarpic eggplant: superior germoplasm for increased winter production. Mol. Breed. 6(1):7986.

11. FAO. 2013. Organización de las Naciones Unidas para la Alimentación y la Agricultura. FAOSTAT. Cultivos. Disponible desde Internet en: http://www.fao.org/ corp/statistics/es/ (con acceso 05/05/2013).

12. FEDERER, W.T. 1961. Augmented designs with one-way elimination of heterogeneity. Biometr. 17:447-473.

13. GAJEWSKI, M.; ARASIMOWICZ, D. 2004. Sensory quality of eggplant fruits (Solanum melongena L.) as affected by cultivar and maturity stage. Pol. J. Food Nutr. Sci. 13/54 (3):249-254.

14. GAJEWSKI, M.; KOWALCZYK, K.; BAJER, M.; RADZANOWSKA, J. 2009. Quality of eggplant fruits in rela- 
tion to growing medium used in greenhouse cultivation and to a cultivar. Not. Bot. Hort. Agrobot. Cluj. 37(1):229-234.

15. GARCÍA, J.A.; MARTÍNEZ, G.; LUJAN, M.; RAMÍREZ, M.; LÓPEZ, A. 2006. Análisis Línea x Probador: Estrategia para selección de progenitores de alto rendimiento en Chile Jalapeño (Capsicum annuum L.). Rev. Agraria -Nueva Época. 3(1,2,3):9-17.

16. HALLAUER, A.; MIRANDA, J.B. 1985. Quantitative Genetics in Maize Breeding. lowa State University Press. 468p.

17. ICONTEC. 2004. Instituto Colombiano de Normas Técnicas. Norma Técnica Colombiana NTC 1220. Berenjena. $4 \mathrm{p}$.

18. KUMAR, S.R.; ARUMUGAN, T.; BALAKRISHNAN, S.; ANANDAKUMAR, C.R. 2013. Variability in the segregating generation of eggplant for earliness and yield. Pakistan J. Biol. Sci. 16(20):1122-1129.

19. MAZZUCATO, A.; FICCADENTI, N.; CAIONI, M.; MOSCONI, P.; PICCININI, E.; REDDY, V.; SESTILI, S.; FERRARI, V. 2010. Genetic diversity and distinctiveness in tomato (Solanum lycopersicum L.) landraces: The Italian case study of 'A pera Abruzzese. Sci. Hort. 125(1):55-62.

20. MOLINA, G.J.D. 1992. Introducción a la genética de poblaciones y cuantitativa. AGT Editor S.A. México D.F. 349p.

21. MORENO, M.M.; PEÑA-LOMELI, A.; SAHAGÚN-CASTELLANOS, J.; RODRÍGUEZ-PÉREZ, J.E.; MORAAGUILAR, R. 2002. Varianza aditiva, heredabilidad y correlaciones en la variedad M1-fitotecnia de tómate de cáscara (Physalisix ocarpa Brot). Rev. Fitotec. Mex. 25(3):231-237.

22. MUNIAPPAN, S.; SARAVANAN, K.; RAMYA, B. 2010. Studies on genetic divergence and variability for certain economic characters in eggplant (Solanum melongena L.). Electr. J. Plant Breed. 1(4):462-465.

23. MUÑOZ-FALCÓN, J.E.; PROHENS, J.; VILANOVA, S.; NUEZ, F. 2009. Diversity in commercial varieties and landraces of black eggplants and implications for broadening the breeders' gen pool. Ann. Appl. Biol. 154(3):453-465.

24. OFOSU-ANIM, J.; OFFEI, S.K.; YAMAKI, S. 2006. Pistil receptivity, pollen tube growth and gene expression during early fruit development in sweet pepper (Capsicum annuum L). Int. J. Agr. Biol. 8(5):576-579.

25. PIRINÇ, V.; PAKYÜREK, A.Y. 2004. A study on comparison of eggplant population with their selfing lines. Int. J. Agr. Biol. 6(5):874-876.

26. POLIGNANO, G.; UGGENT, P.; BISIGNANO, V.; DELLA, C. 2010. Genetic divergence analysis in eggplant (Solanum melongena L.) and allied species. Gen. Res. Crop. Evol. 57(2):171-181.

27. PRABHU, M.; NATARAJAN, S.; PUGALENDHIL, L. 2009a. Genetic parameters in eggplant (Solanum melongena L.) backcross progenies. Am. Euras. J. Sustain. Agr. 3(3):275-279.

28. PRABHU, M.; NATARAJAN, S.; PUGALENDHIL, L. 2009b. Variability and heritability studies in F5 and F6 progenies of brinjal (Solanum melongena L.). Am. Euras. J. Sustain. Agr. 3(3):306-309.

29. PRABHU, M.; NATARAJAN, S. 2008. Correlation and path analysis in brinjal (Solanum melongena L.). Madras Agric. J. 95(1-6):184-187.

30. PROHENS, J.; NUEZ, F. 2001. Variedades tradicionales de berenjena en España. Vida Rural. 46:1-5.

31. RAMESH-BABU, S.; PATIL, R.V. 2008. Characterization and evaluation of brinjal genotypes. Madras Agric. J. 95(1-6):18-23.

32. RYLSKI, I.; NOTHMANN, J.; ARCAN, L. 1984. Differential fertility in short-styled eggplant flowers. Sci. Hort. 22(1):39-46.

33. SAS. Institute Inc. 2008. SAS/STAT® 9.2 User's Guide. Cary, NC: SAS Institute Inc.

34. SAEED, A.; HAYAT, K.; KHAN, A.A.; IQBAL, S.; ABBAS, G. 2007. Assessment of genetic variability and heritability in Lycopersicum esculentum Mill. Int. J. Agr. Biol. 9(2):375-377.

35. SAO, A.; MEHTA, N. 2010. Heterosis in relation to combining ability for yield and quality attributes in Brinjal (Solanum melongena L.). Electr. J. Plant. Breed. 1(4):783-788.

36. SILVA, D.J.H.; COSTA, C.P.; CASALI, V.W.D.; DIAS, L.A.S.; CRUZ, C.D. 1999. Relação entre divergência genética de acessos de berinjela e desempenho de seus híbridos. Hort. Bras. 17(2):129-133. 
37. SINGH, A.K.; PAN, R.S.; RAI, M.; KRISHNA PRASAD, V.S.R. 2003. Genetic studies of quantitative traits in brinjal (Solanum melongena L.). Veg. Sci. 30(2):195197.

38. SUNEETHA, Y.; KATHIRIA, K.B. 2005. Heterosis for yield and yield component characters in round brinjal
(Solanum melongena L.). Madras Agric. J. 92(1012):748-752.

Recibido: Julio 26 de 2013

Aceptado: Mayo 9 de 2014

\section{Como citar:}

Araméndiz-Tatis, H.; Cardona-Ayala, C.; Correa-Álvarez, E. 2014. Parametros genéticos en berenjena (Solanum melongena L.). Rev. U.D.C.A Act. \& Div. Cient. 17(2): 361-369. 